PROCEEDINGS OF THE

AMERICAN MATHEMATICAL SOCIETY

Volume 128, Number 9, Pages 2517-2528

S 0002-9939(00)05555-6

Article electronically published on April 27, 2000

\title{
CLASS NUMBERS \\ OF IMAGINARY ABELIAN NUMBER FIELDS
}

\author{
KU-YOUNG CHANG AND SOUN-HI KWON
}

(Communicated by David E. Rohrlich)

\begin{abstract}
Let $N$ be an imaginary abelian number field. We know that $h_{N}^{-}$, the relative class number of $N$, goes to infinity as $f_{N}$, the conductor of $N$, approaches infinity, so that there are only finitely many imaginary abelian number fields with given relative class number. First of all, we have found all imaginary abelian number fields with relative class number one: there are exactly 302 such fields. It is known that there are only finitely many CMfields $N$ with cyclic ideal class groups of 2-power orders such that the complex conjugation is the square of some automorphism of $N$. Second, we have proved in this paper that there are exactly 48 such fields.
\end{abstract}

\section{INTRODUCTION}

Let $N$ be an imaginary abelian number field of conductor $f_{N}$, maximal real subfield $N^{+}$, and relative class number $h_{N}^{-}$. It is known that $h_{N}^{-}$goes to infinity as $f_{N}$ approaches infinity, so that there exist only finitely many number fields $N$ with $h_{N}^{-}=1$ ([S1, Theorem 2]). It is interesting to find all imaginary number fields with relative class number one. Yamamura has determined all imaginary abelian number fields with class number one. One of the purposes of this paper is to prove the following:

Theorem 1. There are exactly 302 imaginary abelian number fields with relative class number one: 243 out of them are non-cyclic number fields. Their degrees are less than or equal to 24 and their conductors are less than or equal to 65689. These fields are given in Table I.

Louboutin [Lou1] has proved that there are only finitely many CM-fields $N$ with cyclic ideal class groups of 2-power orders such that the complex conjugation is the square of some automorphism of $N$ and he also proved that the relative class numbers of such fields are equal to 1 or 2 . Furthermore he determined the non-quadratic imaginary cyclic number fields of 2-power degrees with cyclic ideal class groups of 2-power orders. Our second goal of this paper is to determine all imaginary abelian number fields with relative class numbers less than or equal to 4 such that the complex conjugation is the square of some automorphism of $N$ :

Received by the editors May 1, 1998.

1991 Mathematics Subject Classification. Primary 11R29; Secondary 11R20.

This research was supported by Grant BSRI-97-1408 from the Ministry of Education of Korea. 
TABLE I. The imaginary non-cyclic abelian number fields with relative class number one. $\chi_{7}(3)=e^{2 \pi i / 6}, \chi_{13}(2)=e^{2 \pi i / 12}, \psi_{9}(2)=$ $e^{2 \pi i / 3}$

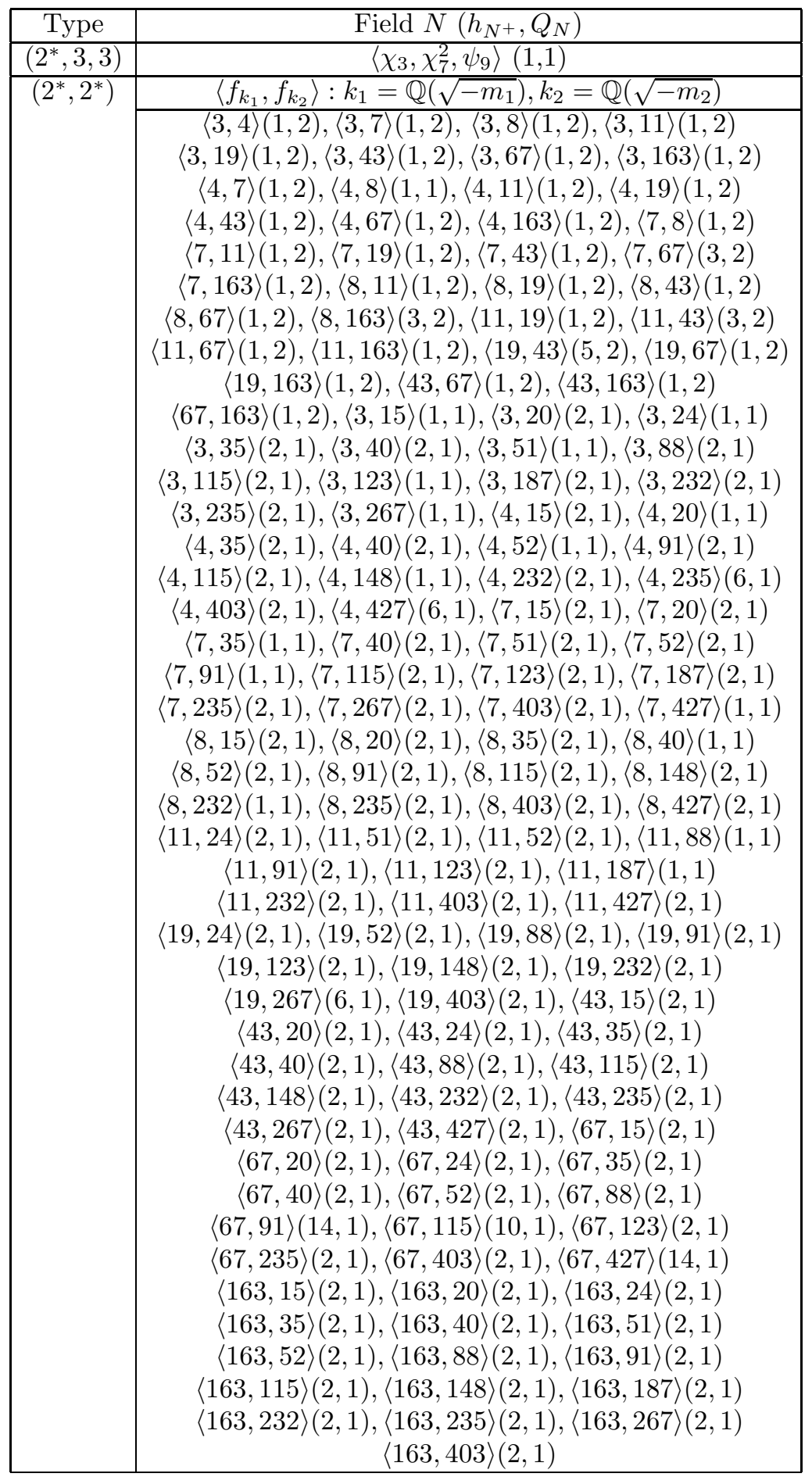




\begin{tabular}{|c|c|}
\hline Type & Field $N\left(h_{N^{+}}, Q_{N}\right)$ \\
\hline$\left(2^{*}, 2^{*}, 3\right)$ & $\begin{array}{c}\left\langle\chi_{7}^{3}, \chi_{3}, \chi_{7}^{2}\right\rangle(1,2),\left\langle\chi_{7}^{3}, \chi_{4}, \chi_{7}^{2}\right\rangle(1,2) \\
\left\langle\chi_{7}^{3}, \chi_{5}^{2} \chi_{7}^{3}, \chi_{7}^{2}\right\rangle(1,1),\left\langle\chi_{7}^{3}, \chi_{4} \psi_{8}, \chi_{7}^{2}\right\rangle(1,2) \\
\left\langle\chi_{7}^{3}, \chi_{11}^{5}, \chi_{7}^{2}\right\rangle(1,2),\left\langle\chi_{7}^{3}, \chi_{3} \chi_{5}^{2}, \chi_{7}^{2}\right\rangle(2,1) \\
\left\langle\chi_{3}, \chi_{4}, \chi_{7}^{2}\right\rangle(1,2),\left\langle\chi_{3}, \chi_{5}^{2} \chi_{7}^{3}, \chi_{7}^{2}\right\rangle(2,1) \\
\left\langle\chi_{3}, \chi_{4} \psi_{8}, \chi_{7}^{2}\right\rangle(1,2),\left\langle\chi_{3}, \chi_{11}^{5}, \chi_{7}^{2}\right\rangle(1,2) \\
\left\langle\chi_{3}, \chi_{3} \chi_{5}^{2}, \chi_{7}^{2}\right\rangle(1,1),\left\langle\chi_{4}, \chi_{5}^{2} \chi_{7}^{3}, \chi_{7}^{2}\right\rangle(2,1) \\
\left\langle\chi_{4}, \chi_{4} \psi_{8}, \chi_{7}^{2}\right\rangle(1,1),\left\langle\chi_{4}, \chi_{11}^{5}, \chi_{7}^{2}\right\rangle(1,2) \\
\left\langle\chi_{4}, \chi_{3} \chi_{5}^{2}, \chi_{7}^{2}\right\rangle(2,1),\left\langle\chi_{5}^{2} \chi_{7}^{3}, \chi_{4} \psi_{8}, \chi_{7}^{2}\right\rangle(2,1) \\
\left\langle\chi_{4} \psi_{8}, \chi_{11}^{5}, \chi_{7}^{2}\right\rangle(3,2),\left\langle\chi_{4} \psi_{8}, \chi_{3} \chi_{5}^{2}, \chi_{7}^{2}\right\rangle(2,1) \\
\left\langle\chi_{3}, \chi_{4}, \psi_{9}\right\rangle(1,2),\left\langle\chi_{3}, \chi_{3} \chi_{5}^{2}, \psi_{9}\right\rangle(1,1) \\
\left\langle\chi_{3}, \chi_{7}^{3}, \psi_{9}\right\rangle(1,2),\left\langle\chi_{3}, \chi_{3} \psi_{8}, \psi_{9}\right\rangle(1,1) \\
\left\langle\chi_{4}, \chi_{3} \chi_{5}^{2}, \psi_{9}\right\rangle(2,1),\left\langle\chi_{4}, \chi_{7}^{3}, \psi_{9}\right\rangle(1,2) \\
\left\langle\chi_{3} \chi_{5}^{2}, \chi_{7}^{3}, \psi_{9}\right\rangle(2,1),\left\langle\chi_{3}, \chi_{7}^{3}, \chi_{13}^{4}\right\rangle(1,2) \\
\left\langle\chi_{3}, \chi_{4} \psi_{8}, \chi_{13}^{4}\right\rangle(1,2),\left\langle\chi_{4} \chi_{13}^{6}, \chi_{7}^{3}, \chi_{13}^{4}\right\rangle(2,1) \\
\left\langle\chi_{4} \chi_{13}^{6}, \chi_{4} \psi_{8}, \chi_{13}^{4}\right\rangle(2,1),\left\langle\chi_{7}^{3}, \chi_{4} \psi_{8}, \chi_{13}^{4}\right\rangle(1,2) \\
\left\langle\chi_{4}, \chi_{19}^{9}, \chi_{19}^{6}\right\rangle(1,2),\left\langle\chi_{3}, \chi_{43}^{21}, \chi_{43}^{14}\right\rangle(1,2) \\
\left\langle\chi_{3}, \chi_{7}^{3}, \chi_{7}^{4} \psi_{9}\right\rangle(3,2),\left\langle\chi_{7}^{3}, \chi_{7}^{3} \chi_{13}^{6}, \chi_{7}^{4} \chi_{13}^{4}\right\rangle(3,1) \\
\end{array}$ \\
\hline$\left(2^{*}, 2^{*}, 5\right)$ & $\left\langle\chi_{3}, \chi_{11}^{5}, \chi_{11}^{2}\right\rangle(1,2),\left\langle\chi_{3}, \chi_{4}, \chi_{11}^{2}\right\rangle(1,2),\left\langle\chi_{4}, \chi_{11}^{5}, \chi_{11}^{2}\right\rangle(1,2)$ \\
\hline$\left(4^{*}, 2^{*}\right)$ & $\begin{array}{c}\left\langle\chi_{5}, \chi_{3}\right\rangle(1,2),\left\langle\chi_{5}, \chi_{4}\right\rangle(1,2),\left\langle\chi_{5}, \chi_{7}^{3}\right\rangle(1,2) \\
\left\langle\chi_{5}, \chi_{4} \psi_{8}\right\rangle(1,2),\left\langle\chi_{13}^{3}, \chi_{4}\right\rangle(1,2),\left\langle\chi_{13}^{3}, \chi_{7}^{3}\right\rangle(1,2) \\
\left\langle\chi_{4} \psi_{16}, \chi_{3}\right\rangle(1,2),\left\langle\chi_{4} \psi_{16}, \chi_{4}\right\rangle(1,1),\left\langle\chi_{4} \psi_{16}, \chi_{11}^{5}\right\rangle(1,2) \\
\left\langle\chi_{4} \psi_{16}, \chi_{4} \chi_{5}^{2}\right\rangle(2,1),\left\langle\chi_{37}^{9}, \chi_{4}\right\rangle(1,2),\left\langle\chi_{29}^{7}, \chi_{4} \psi_{8}\right\rangle(1,2) \\
\left\langle\chi_{4} \psi_{16} \chi_{5}^{2}, \chi_{4}\right\rangle(2,1),\left\langle\chi_{3} \psi_{16}, \chi_{3}\right\rangle(1,1) \\
\left\langle\chi_{3} \psi_{16}, \chi_{11}^{5}\right\rangle(2,1),\left\langle\chi_{7}^{3} \chi_{17}^{4}, \chi_{3}\right\rangle(2,1) \\
\left\langle\chi_{7}^{3} \chi_{17}^{4}, \chi_{11}^{5}\right\rangle(2,1),\left\langle\chi_{61}^{15}, \chi_{7}^{3}\right\rangle(5,2)\end{array}$ \\
\hline$\left(4^{*}, 2^{*}, 3\right)$ & $\left\langle\chi_{5}, \chi_{7}^{3}, \chi_{7}^{2}\right\rangle(1,2),\left\langle\chi_{5}, \chi_{3}, \psi_{9}\right\rangle(1,2),\left\langle\chi_{5}, \chi_{3}, \chi_{7}^{2}\right\rangle(1,2)$ \\
\hline$\left(8^{*}, 2^{*}\right)$ & $\left\langle\chi_{4} \psi_{32}, \chi_{4}\right\rangle(1,1),\left\langle\chi_{4} \psi_{32}, \chi_{3}\right\rangle(1,2),\left\langle\chi_{4} \psi_{32}, \chi_{4} \chi_{5}^{2}\right\rangle(2,1)$ \\
\hline$\left(2^{*}, 2^{*}, 2^{*}\right)$ & $\begin{array}{c}\left(f_{k_{1}}, f_{k_{2}}, f_{k_{3}}\right): k_{1}=\mathbb{Q}\left(\sqrt{-m_{1}}\right), k_{2}=\mathbb{Q}\left(\sqrt{-m_{2}}\right), k_{3}=\mathbb{Q}\left(\sqrt{-m_{3}}\right) \\
\langle 3,4,7\rangle(1,2),\langle 3,4,8\rangle(1,2),\langle 3,4,11\rangle(1,2) \\
\langle 3,4,15\rangle(1,2),\langle 3,4,19\rangle(1,2),\langle 3,7,8\rangle(1,2) \\
\langle 3,7,15\rangle(1,2),\langle 3,8,15\rangle(1,2),\langle 3,11,19\rangle(1,2) \\
\langle 3,11,24\rangle(1,2),\langle 3,11,51\rangle(1,2),\langle 4,7,19\rangle(1,2) \\
\langle 4,7,20\rangle(1,2),\langle 4,7,52\rangle(1,2),\langle 4,8,11\rangle(1,2) \\
\langle 4,8,20\rangle(1,1),\langle 7,8,35\rangle(1,2)\end{array}$ \\
\hline$\left(2^{*}, 2^{*}, 2^{*}, 3\right)$ & $\left\langle\chi_{3}, \chi_{4}, \chi_{7}^{3}, \chi_{7}^{2}\right\rangle(1,2),\left\langle\chi_{3}, \chi_{7}^{3}, \chi_{3} \chi_{5}^{2}, \chi_{7}^{2}\right\rangle(1,2)$ \\
\hline$\left(4^{*}, 2^{*}, 2^{*}\right)$ & $\begin{array}{c}\left\langle\chi_{5}, \chi_{4}, \chi_{4} \psi_{8}\right\rangle(1,2),\left\langle\chi_{5}, \chi_{3}, \chi_{4}\right\rangle(1,2),\left\langle\chi_{5}, \chi_{3}, \chi_{7}^{3}\right\rangle(1,2) \\
\left\langle\chi_{5}, \chi_{3}, \chi_{4} \psi_{8}\right\rangle(1,2),\left\langle\chi_{5}, \chi_{4}, \chi_{7}^{3}\right\rangle(1,2) \\
\left\langle\chi_{4} \psi_{16}, \chi_{3}, \chi_{4}\right\rangle(1,2),\left\langle\chi_{4} \psi_{16}, \chi_{4}, \chi_{4} \chi_{5}^{2}\right\rangle(1,1)\end{array}$ \\
\hline$\left(4^{*}, 2\right)$ & $\begin{array}{c}\left\langle\chi_{5}, \psi_{8}\right\rangle(1,1),\left\langle\chi_{5}, \chi_{13}^{6}\right\rangle(1,1),\left\langle\chi_{5}, \chi_{17}^{8}\right\rangle(1,1) \\
\left\langle\chi_{13}^{3}, \chi_{5}^{2}\right\rangle(1,1),\left\langle\chi_{13}^{3}, \psi_{8}\right\rangle(1,1),\left\langle\chi_{4} \psi_{16}, \chi_{5}^{2}\right\rangle(1,1)\end{array}$ \\
\hline$\left(4^{*}, 4^{*}\right)$ & $\left\langle\chi_{5}, \chi_{13}^{3}\right\rangle(1,2),\left\langle\chi_{5}, \chi_{4} \psi_{16}\right\rangle(1,2)$ \\
\hline
\end{tabular}

Theorem 2. There are exactly 107 imaginary abelian number fields with relative class numbers $\leq 4$ such that the complex conjugation is the square of some automorphism of $N: 58$ out of them are non-cyclic number fields. These fields are given in Table II. 
TABLE II. The imaginary non-cyclic abelian number fields with relative class number 2 and 4 . The fields with non-trivial cyclic ideal class group of 2-power orders are mentioned with $\langle,\rangle^{c}$.

\begin{tabular}{|c|c|c|}
\hline Type & $h_{N}^{-}$ & Field $N\left(h_{N^{+}}, Q_{N}\right)$ \\
\hline \multirow[t]{2}{*}{$\left(4^{*}, 2\right)$} & 2 & $\begin{array}{c}\left\langle\chi_{4} \psi_{16}, \chi_{3} \chi_{4}\right\rangle^{c}(1,2),\left\langle\chi_{5}, \chi_{3} \chi_{4}\right\rangle^{c}(1,1) \\
\left\langle\chi_{5}, \chi_{3} \chi_{7}^{3}\right\rangle^{c}(1,1),\left\langle\chi_{5}, \chi_{3} \chi_{4} \psi_{8}\right\rangle^{c}(1,1) \\
\left\langle\chi_{5}, \chi_{4} \chi_{7}^{3}\right\rangle^{c}(1,1),\left\langle\chi_{5}, \chi_{29}^{14}\right\rangle^{c}(2,1) \\
\left\langle\chi_{13}^{3}, \chi_{17}^{8}\right\rangle^{c}(1,1),\left\langle\chi_{29}^{7}, \chi_{5}^{2}\right\rangle^{c}(2,1) \\
\left\langle\chi_{5} \psi_{8}, \psi_{8} \chi_{13}^{6}\right\rangle^{c}(4,1),\left\langle\chi_{5} \psi_{8}, \psi_{8} \chi_{17}^{8}\right\rangle^{c}(2,1) \\
\left\langle\chi_{5} \chi_{13}^{6}, \chi_{13}^{6} \chi_{17}^{8}\right\rangle^{c}(2,1),\left\langle\chi_{5}^{2} \chi_{13}^{3}, \chi_{5}^{2} \psi_{8}\right\rangle^{c}(4,1) \\
\left\langle\chi_{3} \psi_{16}, \chi_{3} \chi_{4} \chi_{5}^{2}\right\rangle(2,1)\end{array}$ \\
\hline & 4 & $\begin{array}{c}\left\langle\chi_{4} \psi_{16}, \chi_{4} \chi_{7}^{3}\right\rangle(1,2),\left\langle\chi_{4} \psi_{16}, \chi_{17}^{8}\right\rangle(1,1) \\
\left\langle\chi_{3} \psi_{16}, \chi_{3} \chi_{7}^{3}\right\rangle(1,1),\left\langle\chi_{4} \psi_{16} \chi_{5}^{2}, \chi_{4} \chi_{5}^{2} \chi_{7}^{3}\right\rangle(2,1) \\
\left\langle\chi_{5}, \chi_{3} \chi_{11}^{5}\right\rangle(1,1),\left\langle\chi_{5}, \chi_{41}^{20}\right\rangle(1,1) \\
\left\langle\chi_{5}, \chi_{4} \chi_{11}^{5}\right\rangle(1,1),\left\langle\chi_{5}, \chi_{3} \chi_{19}^{9}\right\rangle(1,1) \\
\left\langle\chi_{5}, \chi_{61}^{30}\right\rangle(1,1),\left\langle\chi_{13}^{3}, \chi_{3} \chi_{4}\right\rangle(1,1) \\
\left\langle\chi_{13}^{3}, \chi_{3} \chi_{7}^{3}\right\rangle(1,1),\left\langle\chi_{13}^{3}, \chi_{29}^{14}\right\rangle(1,1) \\
\left\langle\chi_{5} \psi_{8}, \chi_{3} \chi_{4} \psi_{8}\right\rangle(1,1),\left\langle\chi_{5} \psi_{8}, \chi_{3} \chi_{7}^{3} \psi_{8}\right\rangle(2,1) \\
\left\langle\chi_{5} \psi_{8}, \chi_{3} \chi_{4}\right\rangle(1,1),\left\langle\chi_{5} \psi_{8}, \chi_{4} \psi_{8} \chi_{7}^{3}\right\rangle(1,1) \\
\left\langle\chi_{5} \psi_{8}, \psi_{8} \chi_{29}^{14}\right\rangle(4,1),\left\langle\chi_{5} \chi_{13}^{6}, \chi_{3} \chi_{4} \chi_{13}^{6}\right\rangle(2,1) \\
\left\langle\chi_{5} \chi_{13}^{6}, \chi_{3} \chi_{7}^{3} \chi_{13}^{6}\right\rangle(2,1),\left\langle\chi_{5} \chi_{13}^{6}, \chi_{3} \chi_{4} \psi_{8} \chi_{13}^{6}\right\rangle(2,1) \\
\left\langle\chi_{5} \chi_{13}^{6}, \chi_{4} \chi_{7}^{3} \chi_{13}^{6}\right\rangle(4,1),\left\langle\chi_{5} \chi_{13}^{6}, \chi_{13}^{6} \chi_{29}^{14}\right\rangle(4,1) \\
\left\langle\chi_{5} \chi_{17}^{8}, \chi_{3} \chi_{4} \chi_{17}^{8}\right\rangle(2,1),\left\langle\chi_{5} \chi_{17}^{8}, \chi_{3} \chi_{7}^{3} \chi_{17}^{8}\right\rangle(8,1) \\
\left\langle\chi_{5} \chi_{17}^{8}, \chi_{3} \chi_{4} \psi_{8} \chi_{17}^{8}\right\rangle(2,1),\left\langle\chi_{5} \chi_{17}^{8}, \chi_{4} \chi_{7}^{3} \chi_{17}^{8}\right\rangle(2,1) \\
\left\langle\chi_{5} \chi_{17}^{8}, \chi_{17}^{8} \chi_{29}^{14}\right\rangle(4,1),\left\langle\chi_{5}^{2} \chi_{13}^{3}, \chi_{5}^{2} \chi_{17}^{8}\right\rangle(2,1) \\
\left\langle\chi_{13}^{3} \psi_{8}, \psi_{8} \chi_{17}^{8}\right\rangle(4,1),\left\langle\chi_{7}^{3} \chi_{17}^{4}, \chi_{4} \chi_{7}^{3}\right\rangle(1,1) \\
\left\langle\chi_{7}^{3} \chi_{17}^{4}, \chi_{4} \psi_{8} \chi_{7}^{3}\right\rangle(1,1),\left\langle\chi_{7}^{3} \chi_{17}^{4}, \chi_{3} \chi_{5}^{2} \chi_{7}^{3}\right\rangle(8,1) \\
\end{array}$ \\
\hline$\left(4^{*}, 2,3\right)$ & 4 & $\left\langle\chi_{13}^{3}, \chi_{5}^{2}, \chi_{13}^{4}\right\rangle(1,1)$ \\
\hline$\left(8^{*}, 2\right)$ & 4 & $\left\langle\chi_{3} \chi_{17}^{2}, \chi_{3} \chi_{4}\right\rangle(1,1)$ \\
\hline$\left(4^{*}, 4^{*}\right)$ & 4 & $\left\langle\chi_{5}, \chi_{29}^{7}\right\rangle(2,2),\left\langle\chi_{4} \psi_{16}, \chi_{3} \chi_{4} \chi_{5}\right\rangle(2,1)$ \\
\hline$\left(4^{*}, 2,2\right)$ & 4 & $\left\langle\chi_{5}, \psi_{8}, \chi_{3} \chi_{4}\right\rangle(1,1)$ \\
\hline
\end{tabular}

Corollary 1. There are exactly 48 imaginary non-quadratic abelian number fields $N$ with cyclic ideal class groups of 2-power orders such that the complex conjugation is the square of some automorphism of $N$ : 20 out of them are non-cyclic number fields. Their class numbers are less than or equal to 8.

This paper is organized as follows. Section 2 reviews some of the standard facts on imaginary abelian number fields. In Section 3 we briefly sketch our method of computations. Throughout this paper the following notations will be used. For a number field $K$, let $O_{K}, C_{K}, d_{K}, h_{K}$ and $\zeta_{K}$ be the ring of integers, the ideal class group, the absolute value of discriminant, the class number and Dedekind zeta function of $K$, respectively. If $K$ is abelian, let us denote by $f_{K}$ the conductor of $K$. If $K$ is a CM-field, we will denote by $K^{+}, h_{K}^{-}, \omega_{K}, Q_{K}$ the maximal real subfield, the relative class number, the number of roots of unity in $K$ and the Hasse unit index of $K$, respectively. For an odd prime $p$ let $\chi_{p}$ be an odd Dirichlet character of conductor $p$, order $p-1$. For $\rho \geq 2$, let $\psi_{p^{\rho}}$ be an even primitive Dirichlet character of conductor $p^{\rho}$, order $p^{\rho-1}$ with $\psi_{p^{\rho}}^{p}=\psi_{p^{\rho-1}}$. For the prime 2 , let $\chi_{4}$ be the odd 
Dirichlet quadratic character of conductor 4 . When $\rho \geq 3$, let $\psi_{2^{\rho}}$ be the even primitive Dirichlet character of conductor $2^{\rho}$, order $2^{\rho-2}$ with $\psi_{2^{\rho}}^{2}=\psi_{2^{\rho-1}}$.

\section{Preliminaries}

In this section we sum up some of the standard facts on imaginary abelian number fields which will be used in the sequel.

Proposition 1. (1) Let $F$ be an imaginary abelian number field, $\chi_{F}$ the group of primitive Dirichlet characters associated to $F$ and $\chi_{F}^{-}$the set of $\chi \in \chi_{F}$ such that $\chi(-1)=-1$. For a $\chi \in \chi_{F}$ we denote by $f_{\chi}$ the conductor of $\chi$. We have

$$
h_{F}^{-}=Q_{F} \omega_{F} \prod_{\chi \in \chi_{F}^{-}}\left(-\frac{1}{2} B_{1, \chi}\right),
$$

where $B_{1, \chi}=\sum_{a=1}^{f_{\chi}-1} \chi(a) a / f_{\chi}$.

(2) Let $K \subset L$ be two $C M$-fields. Then $h_{K}^{-}$divides $4 h_{L}^{-}$. In addition, if $[L: K]$ is odd, then $h_{K}^{-}$divides $h_{L}^{-}$.

Proof. (1) is the content of [W, Theorem 4. 17]. (2) For the first statement see [OK. Theorem 1] and [Ho, Theorem 5]. For the second statement see [Lem, Corollary 1] or [LOO, Theorem 5].

Proposition 2. Let $K$ be a CM-field of degree $2 n$.

(1) We have

$$
h_{K}^{-}=\frac{Q_{K} \omega_{K}}{(2 \pi)^{n}} \sqrt{\frac{d_{K}}{d_{K^{+}}}} \frac{\operatorname{Res}_{s=1}\left(\zeta_{K}\right)}{\operatorname{Res}_{s=1}\left(\zeta_{K^{+}}\right)} .
$$

(2) The fact that $\beta \in\left[1-2 / \log d_{K}, 1\left[\right.\right.$ and $\zeta_{K}(\beta) \leq 0$ implies

$$
\begin{array}{r}
\operatorname{Res}_{s=1}\left(\zeta_{K}\right) \geq \frac{\varepsilon_{K}}{e}(1-\beta), \\
\text { where } \varepsilon_{K}=\max \left(1-\frac{2 \pi n e^{1 / n}}{d_{K}^{1 / 2 n}}, \frac{2}{5} \exp \left(-\frac{2 \pi n}{d_{K}^{112 n}}\right)\right) .
\end{array}
$$

(3) (a) There exists a constant $\mu_{k}>0$ such that for any abelian extension $K / k$ of degree $m$ unramified at all the infinite places we have

$$
\operatorname{Res}_{s=1}\left(\zeta_{K}\right) \leq\left(\operatorname{Res}_{s=1}\left(\zeta_{k}\right)\right)^{m}\left(\frac{1}{2(m-1)} \log \left(\frac{d_{K}}{d_{k}^{m}}\right)+2 \mu_{k}\right)^{m-1} .
$$

(b) If $k$ is a real abelian number field of degree $s \geq 2$, then

$$
\begin{gathered}
\mu_{k} \operatorname{Res}_{s=1}\left(\zeta_{k}\right) \leq \frac{s-1}{2^{s+1}}\left(\log f_{k}+2 \mu_{\mathbb{Q}}\right)^{s}, \\
\text { where } \mu_{\mathbb{Q}}=(2+\gamma-\log (4 \pi)) / 2, \gamma \text { is Euler's constant. }
\end{gathered}
$$

Proof. (1) is the content of [W, Chapter 4]. (2) See [Lou2, Proposition A] or [LO, Proposition 9]. (3) is the content of Theorem 1, Corollary 2 and Theorem 11 in Lou3.

Proposition 3 ([Lou4 Lemma (b)]). Let $K$ be an imaginary cyclic number field of degree $2^{n}, n \geq 1$. For a positive integer $n$ we let $\zeta_{n}$ be a primitive nth root of unity. Then, $\omega_{K}=2$, except when $K=\mathbb{Q}\left(\zeta_{4}\right)$ (in which case $\left.\omega_{K}=4\right)$, or when $2^{n}+1$ is prime and $K=\mathbb{Q}\left(\zeta_{2^{n}+1}\right)$ (in which case $\omega_{K}=2\left(2^{n}+1\right)$ ). 
Proposition 4. Let $L$ be a CM-field. We denote by $i_{L / L^{+}}$the homomorphism $C_{L^{+}} \rightarrow C_{L}$ induced by mapping an ideal $\mathfrak{a}$ to $\mathfrak{a} O_{L}$. We write $L=L^{+}(\sqrt{\alpha})$ for some $\alpha \in O_{L^{+}}$.

(1) ([Ha, Satz 24]) If $L$ is a cyclic number field, then $Q_{L}=1$.

(2) $\left(\left[\mathrm{Lem}\right.\right.$, Theorem 1]) Assume that $\omega_{L} \equiv 2 \bmod 4$.

(a) If the principal ideal $(\alpha)$ is not a square of an ideal of $O_{L^{+}}$, then $Q_{L}=1$ and $i_{L / L^{+}}$is injective.

(b) Assume that there is an ideal $\mathfrak{b}$ in $O_{L^{+}}$such that $(\alpha)=\mathfrak{b}^{2}$.

i) $Q_{L}=2$, if $\mathfrak{b}$ is principal.

ii) $Q_{L}=1$ and $\operatorname{ker}\left(i_{L / L^{+}}\right)=\langle[\mathfrak{b}]\rangle$, otherwise.

Proposition 5. Let $N$ be an imaginary cyclic number field.

(1) If $[N: \mathbb{Q}]$ is a power of 2 and if $h_{N}^{-} \leq 16$, then $[N: \mathbb{Q}] \leq 16$.

(2) If $h_{N}^{-} \leq 4$, then $[N: \mathbb{Q}] \leq 22$.

Proof. (1) See [PK2]. (2) See CK].

\section{MAIN RESULTS}

Let $s$ be a positive integer, $r$ a non-negative integer. For $1 \leq i \leq s$ let $m_{i}$ be a positive integer, for $1 \leq j \leq r$ let $n_{j}$ be a power of a prime number. Assume that $m_{1} \geq m_{2} \geq \cdots \geq m_{s}$ and $n_{j}<2^{m_{s}}$ for each $j$ such that $n_{j}$ is a power of 2 . The imaginary abelian field $N$ is called of type $\left(2^{m_{1}^{*}}, \cdots, 2^{m_{s}^{*}}, n_{1}, \cdots, n_{r}\right)$ if $N$ is a compositum of $s$ imaginary cyclic number fields $K_{i}$ of degree $2^{m_{i}}, 1 \leq i \leq s$, and $r$ real cyclic number fields $L_{j}$ of degree $n_{j}, 1 \leq j \leq r$, such that $K_{i+1} \cap\left(K_{1} \cdots K_{i}\right)=\mathbb{Q}$ for each $i=1, \cdots, s-1$ and $L_{j+1} \cap\left(K_{1} \cdots K_{s} L_{1} \cdots L_{j}\right)=\mathbb{Q}$ for each $j=0, \cdots$, $r-1$. Let $G$ be the Galois group of $N$ over $\mathbb{Q}$. Then $G$ is isomorphic to the direct product $\prod_{i=1}^{s} \mathbb{Z} / 2^{m_{i}} \mathbb{Z} \prod_{j=1}^{r} \mathbb{Z} / n_{j} \mathbb{Z}$.

This section is divided into subsections:

3.1. Proof of Theorem 1.

3.2. Proof of Theorem 2.

3.3. Proof of Corollary 1.

3.1. Proof of Theorem 1. In order to determine all imaginary abelian number fields with relative class number one we proceed as follows. Every imaginary abelian number field with relative class number one is a compositum of a finite number of distinct imaginary cyclic number fields with relative class number 1 , 2 , or 4 . Since all imaginary cyclic number fields with relative class number $\leq 4$ are known, there remain only finitely many computations to determine all imaginary abelian number fields with relative class number one ([S2], [S3], [A], [MW], [Lou5], PK1], PK2], and [CK]). Assume that $N$ is an imaginary abelian number field of type $\left(2^{m_{1}^{*}}, \cdots, 2^{m_{s}^{*}}, n_{1}, \cdots, n_{r}\right)$ with relative class number one. A primitive Dirichlet character will be called a character in brief. Let $\tau_{1}, \cdots, \tau_{s}$ be odd characters of order $2^{m_{i}}, 1 \leq i \leq s$, and let $\varphi_{1}, \cdots, \varphi_{r}$ be even characters of order $n_{j}, 1 \leq j \leq r$, such that $N$ is associated with the group $\left\langle\tau_{1}, \cdots, \tau_{s}, \varphi_{1}, \cdots, \varphi_{r}\right\rangle$. Since $h_{N}^{-}=1$, the subfields $M_{i}$ associated with $\left\langle\tau_{i}\right\rangle, 1 \leq i \leq s$, satisfy that $h_{M_{i_{1}} \cdots M_{i_{a}}}^{-} \mid 4$ for $1 \leq a \leq s, i_{1}, \cdots, i_{a} \in\{1, \cdots, s\}$. If $n_{j}$ is odd, then the cyclic subfields $L_{i}$ associated with $\left\langle\tau_{i}, \varphi_{j}\right\rangle, 1 \leq i \leq s$, satisfy $h_{L_{i}}^{-} \mid 4$. If $n_{j}$ is a power of $2, n_{j}=2^{m}$, then the relative class numbers of the cyclic subfields associated with $\left\langle\tau_{i} \varphi_{j}^{k}\right\rangle, 1 \leq i \leq s, 1 \leq k \leq 2^{m}$, divide 4 . On the other hand, if there is no field 
of type $\left(2^{m_{1}^{*}}, 2^{m_{2}^{*}}, \cdots, 2^{m_{s}^{*}}, n_{1}, \cdots, n_{r}\right)$ with relative class number dividing 4 such that all imaginary subfields have relative class number dividing 4 , then we conclude that there is no field with relative class number one containing a subfield of type $\left(2^{m_{1}^{*}}, \cdots, 2^{m_{s}^{*}}, n_{1}, \cdots, n_{r}\right)$. For the fields $N$ of type $\left(2^{*}, 2^{*}\right)$ or $\left(2^{*}, 2^{*}, 2^{*}\right)$ we determine $Q_{N}$ using [HY1], [HY2] and [HY3]. For other fields we use Proposition 4. In order to determine whether an ideal $\mathfrak{b}$ is principal or not we use the function IdealIsPrincipal in KASH [KT]. By Proposition 1.(1) we can easily evaluate $h_{N}^{-}$. Our computational results are summarized in the following. All fields mentioned below are given in Tables I and II.

Proposition 6. Let $l$ be an odd prime number.

(1) (a) There is only one field of type $\left(2^{*}, 3,3\right)$ with relative class number one: this field is associated with $\left\langle\chi_{3}, \chi_{7}^{2}, \psi_{9}\right\rangle$ and has class number one.

(b) There is no field $M$ with relative class number one such that $M$ contains a subfield of type $\left(2^{*}, 3,3,3\right)$ or a subfield of type $\left(2^{*}, 3,9\right)$.

(2) If $l \geq 5$, then there is no field with relative class number one in which a field of type $\left(2^{*}, l, l\right)$ is contained.

(3) If $l \geq 3$, then there is no field with relative class number one in which a field of type $\left(4^{*}, l, l\right)$ is contained ( $c f$. Proposition 15 below).

Proposition 7. (1) (a) There are exactly 147 fields of type $\left(2^{*}, 2^{*}\right)$ with relative class number one.

(b) There are exactly 34 fields of type $\left(2^{*}, 2^{*}, 3\right)$ with relative class number one.

(c) There is no field with relative class number one in which a field of type $\left(2^{*}, 2^{*}, 3,3\right)$ or type $\left(2^{*}, 2^{*}, 9\right)$ is contained.

(2) (a) There are 3 fields of type $\left(2^{*}, 2^{*}, 5\right)$ with relative class number one.

(b) There is no field with relative class number one in which a field of type $\left(2^{*}, 2^{*}, 5,5\right)$ is contained.

(3) Let $l$ be an odd prime number $\geq 7$. There is no field with relative class number one in which a field of type $\left(2^{*}, 2^{*}, l\right)$ is contained.

Proposition 8. (1) There are 18 fields of type $\left(4^{*}, 2^{*}\right)$ with relative class number one.

(2) There are 3 fields of type $\left(4^{*}, 2^{*}, 3\right)$ with relative class number one.

(3) There is no field with relative class number one containing a subfield of type $\left(4^{*}, 2^{*}, 5\right)$.

Proposition 9. (1) There are three fields of type $\left(8^{*}, 2^{*}\right)$ with relative class number one.

(2) Let $l$ be an odd prime number. There is no field with relative class number one containing a subfield of type $\left(8^{*}, 2^{*}, l\right)$.

Proposition 10. There is no field with relative class number one containing a field of type $\left(16^{*}, 2^{*}\right)$.

Proposition 11. (1) There are 6 fields of type $\left(4^{*}, 2\right)$ with relative class number one.

(2) Let l be a prime. There is no field with relative class number one containing a subfield of type $\left(4^{*}, 2, l\right)$.

(3) There is no field with relative class number one containing a subfield of type $\left(8^{*}, 2\right)$. 
(4) There is no field with relative class number one containing a subfield of type $\left(16^{*}, 2\right)$ (cf. Proposition 19).

(5) There are 2 fields of type $\left(4^{*}, 4^{*}\right)$ with relative class number one.

Proposition 12. (1) There are 17 fields of type $\left(2^{*}, 2^{*}, 2^{*}\right)$ with relative class number one.

(2) There are two fields of type $\left(2^{*}, 2^{*}, 2^{*}, 3\right)$ with relative class number one.

(3) There is no field with relative class number one containing a subfield of type $\left(2^{*}, 2^{*}, 2^{*}, l\right)$ for every prime $l \geq 5$.

Proposition 13. (1) There are 7 fields of type $\left(4^{*}, 2^{*}, 2^{*}\right)$ with relative class number one.

(2) There is no field with relative class number one containing a subfield of type $\left(4^{*}, 2^{*}, 2^{*}, l\right)$ for every odd prime $l$.

Proposition 14. There is no field with relative class number one containing a subfield of type $\left(2^{*}, 2^{*}, 2^{*}, 2^{*}\right)$.

Theorem 1 follows from Propositions 6-14.

\subsection{Proof of Theorem 2.}

Lemma 1. Let $N$ be of type $\left(2^{m_{1}^{*}}, \cdots, 2^{m_{s}^{*}}, n_{1}, \cdots, n_{s}\right)$. The complex conjugation is the square of some automorphism of $N$ if and only if $m_{1} \geq \cdots \geq m_{s} \geq 2$.

Proof. Clear.

According to [PK2, Proposition 3] it is clear that there is no field $N$ of type $\left(2^{m_{1}^{*}}, \cdots, 2^{m_{s}^{*}}, n_{1}, \cdots, n_{s}\right)$ with relative class number 3 if $m_{1} \geq \cdots \geq m_{s} \geq 2$. The content of Theorem 2 is divided into Proposition 15-19 according to the Galois groups. Note that there are 8 fields of type $\left(4^{*}, 3\right)$ with relative class number $\leq 4$ : 6 of them have relative class number 1 and 2 of them have relative class number 4. There is exactly one field of type $\left(4^{*}, 5\right)$ with relative class number $\leq 4: \mathbb{Q}\left(\zeta_{25}\right)$. For an odd prime $l$ with $l \geq 7$, there is no field of type $\left(4^{*}, l\right)$ with relative class number $\leq 4$ (see [CK]).

Proposition 15. Let $l$ be an odd prime.

(1) There is no field $N$ with relative class number dividing 4 such that $N$ contains a subfield of type $\left(4^{*}, l, l\right)$.

(2) There is no field $N$ with relative class number dividing 4 such that $N$ contains a subfield of type $\left(8^{*}, l\right)$.

Proof. (1) Let $M$ be a number field of type $\left(4^{*}, l, l\right), \varphi$ an odd character of order 4 , $\chi_{1}, \chi_{2}$ two characters of order $l$ such that $M$ is associated with the group $\left\langle\varphi, \chi_{1}, \chi_{2}\right\rangle$. Suppose that there exists a field $N$ containing $M$ with $h_{N}^{-} \mid 4$. By Proposition 1.(2) all subfields of degree $4 l$ associated with $\left\langle\varphi, \chi_{1}^{i} \chi_{2}^{j}\right\rangle, 1 \leq i, j \leq l$ and $i+j \lesseqgtr 2 l$, have relative class number dividing 4 . Using the results in [PK2] and CK] we verify that there is no such characters $\varphi, \chi_{1}, \chi_{2}$. From this we deduce (1).

(2) is proved similarly as (1).

Proposition 16. (1) There are 13 fields of type $\left(4^{*}, 2\right)$ with relative class number 2 and 32 fields of type $\left(4^{*}, 2\right)$ with relative class number 4.

(2) There is no field of type $\left(4^{*}, 2,3\right)$ with relative class number 2 and there is one field of type $\left(4^{*}, 2,3\right)$ with relative class number 4 . 
(3) There is no field with relative class number $\leq 4$ containing a subfield of type $\left(4^{*}, 2, l\right)$, where $l$ is an odd prime $\geq 5$.

(4) There is no field with relative number $\leq 4$ containing a subfield of type $\left(4^{*}, 2,9\right)$.

(5) There is no field of type $\left(4^{*}, 2,2\right)$ with relative class number 2. There is one field of type $\left(4^{*}, 2,2\right)$ with relative class number 4 .

(6) There is no field with relative class number $\leq 4$ containing a subfield of type $\left(4^{*}, 2,2, l\right)$, where $l$ is a prime.

Proof. (1) Let $N$ be a number field of type $\left(4^{*}, 2\right), \varphi$ an odd character of order 4 and $\chi$ an even quadratic character such that $N$ is associated with $\langle\varphi, \chi\rangle$. Let $M_{1}$ and $M_{2}$ be the quartic subfield associated with $\langle\varphi\rangle$ and $\langle\varphi \chi\rangle$, respectively. Assume that $h_{N}^{-} \mid 4$. There are exactly 133 pairs of $\langle\varphi, \varphi \chi\rangle$ such that $h_{M_{i}}^{-} \mid 8, i=1$ and 2. It is sufficient to consider $M_{i}$ with $h_{M_{i}}^{-} \mid 8$, instead of those with $h_{M_{i}}^{-} \mid 16$, since $h_{N}^{-}=\frac{Q_{N}}{2} h_{M_{1}}^{-} h_{M_{2}}^{-}$. For these fields we determine $Q_{N}$ and $h_{N}^{-}$. Note that if $h_{N}^{-} \mid 4$, then $f_{\varphi^{2}} \in\{5,8,13,17,29\}$ where $f_{\varphi^{2}}$ is the conductor of $\varphi^{2}$. We will need this remark in 2.(a) below.

(2) The computation consists of two steps : (a) Determine all imaginary cyclic number fields of degree 12 with relative class numbers dividing 16 such that its quartic subfield can be embedded into a field $N$ of type $\left(4^{*}, 2\right)$ with $h_{N}^{-} \mid 4$. (b) We consider the composite of $N$ and $K$, where $N$ is one of the fields found in (1), $K$ one of those obtained in (a).

(a) Let $K$ be an imaginary cyclic number field of degree $12, L$ the quartic subfield and $k$ the quadratic subfield of $K$. Assume that $h_{K}^{-} \mid 16$ and $L$ can be embedded into a field $N$ of type $\left(4^{*}, 2\right)$ with $h_{N}^{-} \mid 4$. Using Proposition 2 we find an upper bound for $f_{K}$. According to (1) $f_{k} \in\{5,8,13,17,29\}$. For those quadratic fields $k$ we verify that $\zeta_{k}(s) \leq 0$ for $\left.s \in\right] 0,1\left[\right.$. Note that $\zeta_{K} / \zeta_{k}$ is a product of L-functions which come in conjugate pairs. Hence $\zeta_{K}(s) \leq 0$ for $\left.s \in\right] 0,1[$. By Proposition (2.2) we have

$$
\operatorname{Res}_{s=1}\left(\zeta_{K}\right) \geq \frac{\varepsilon_{K}}{e} \frac{2}{\log d_{K}} .
$$

Since $f_{K}^{6} \leq d_{K} \leq f_{K}^{11}([\mathrm{Mu}$, Corollary 1], [HH Lemma 10] or [T, Lemma 1]), we have

$$
\begin{aligned}
& h_{K}^{-}=\frac{Q_{K} \omega_{K}}{(2 \pi)^{6}} \sqrt{\frac{d_{K}}{d_{K^{+}}}} \frac{\operatorname{Res}_{s=1}\left(\zeta_{K}\right)}{\operatorname{Res}_{s=1}\left(\zeta_{K^{+}}\right)} \\
& \geq \frac{2 \omega_{K} \eta_{K}}{(2 \pi)^{6}} \frac{f_{L} f_{K}^{2}}{e \cdot 11 \log f_{K}} \frac{1}{\operatorname{Res}_{s=1}\left(\zeta_{K^{+}}\right)},
\end{aligned}
$$

where $\eta_{K}=\max \left(1-\frac{12 \pi e^{1 / 6}}{\sqrt{f_{K}}}, \frac{2}{5} \exp \left(-\frac{12 \pi}{\sqrt{f_{K}}}\right)\right)$.

According to Proposition 2.(3),

$$
\operatorname{Res}_{s=1}\left(\zeta_{K^{+}}\right) \leq \operatorname{Res}_{s=1}\left(\zeta_{k}\right)\left(\frac{1}{4} \operatorname{Res}_{s=1}\left(\zeta_{k}\right) \log \frac{d_{K^{+}}}{d_{k}^{3}}+2 \mu_{k} \operatorname{Res}_{s=1}\left(\zeta_{k}\right)\right)^{2}
$$

and

$$
\mu_{k} \operatorname{Res}_{s=1}\left(\zeta_{k}\right) \leq \frac{1}{8}\left(\log f_{k}+2 \times 0.0231\right)^{2} .
$$

Computing $\operatorname{Res}_{s=1}\left(\zeta_{k}\right)$ for these 5 quadratic fields $k$ we verify that $f_{K} \leq 18000$. For such fields $K$ with $f_{K} \leq 18000$ we compute $h_{K}^{-}$and verify that there are 4 
fields $K$ with $h_{K}^{-}=1,2$ fields $K$ with $h_{K}^{-}=4$ and 2 fields $K$ with $h_{K}^{-}=8$. There is no field $K$ with $h_{K}^{-}=16$.

(b) Let $E$ be a number field of type $\left(4^{*}, 2,3\right), \varphi$ an odd character of order $4, \chi$ an even quadratic character and $\tau$ a character of order 3 such that $E$ is associated with the group $\langle\varphi, \chi, \tau\rangle$. Let $N$ be the subfield associated with $\langle\varphi, \chi\rangle$, and $K_{1}$ and $K_{2}$ those associated with $\langle\varphi, \tau\rangle$ and $\langle\varphi \chi, \tau\rangle$, respectively. If $h_{E}^{-} \mid 4$, then $N$ is one of the fields obtained in (1) and $K_{i}, i=1$ and 2, is one of those obtained in (a). There are only two fields $E$ of which the subfields satisfy the above conditions: the fields associated with $\left\langle\chi_{5}, \chi_{13}^{6}, \chi_{13}^{4}\right\rangle$ and $\left\langle\chi_{13}^{3}, \chi_{5}^{2}, \chi_{13}^{4}\right\rangle$, respectively. The former has relative class number 16 and the latter has relative class number 4 .

Similarly, we get (3), (4), (5) and (6).

By the same reasoning as Proposition 16 we get the following.

Proposition 17. (1) There is no field of type $\left(4^{*}, 4^{*}\right)$ with relative class number 2 and two fields with relative class number 4.

(2) There is no field with relative class number $\leq 4$ containing a subfield of type $\left(4^{*}, 4^{*}, l\right), l$ a prime.

Proposition 18. (1) There is no field of type $\left(8^{*}, 2\right)$ with relative class number 2 and one field with relative class number 4.

(2) There is no field with relative class number $\leq 4$ containing a subfield of type $\left(8^{*}, 2, l\right), l$ prime.

(3) There is no field with relative class number $\leq 4$ containing a subfield of type $\left(8^{*}, 4^{*}\right)$ or type $\left(8^{*}, 4\right)$.

Proposition 19. There is no field with relative class number $\leq 4$ containing a subfield of type $\left(16^{*}, l\right), l$ prime.

3.3. Proof of Corollary 1. According to Lou1, Theorem 2], if an imaginary non-quadratic abelian number field $N$ such that the complex conjugation is the square of some automorphism of $N$ has cyclic ideal class group of 2-power order, then $h_{N}^{-}=1$ or 2 . The class numbers of the maximal real subfield of the fields in Theorems 1 and 2 are obtained from [K], G], Li], [Y1] and [KT]. (We note that [KT] is used only for the fields of type $(4,2)$.) Therefore, we verify that among the fields in Theorem 2 there are 21 imaginary non-cyclic number fields $N$ such that

(i) $h_{N}^{-}=1$ or 2 ,

(ii) $h_{N^{+}}=2^{a}$ for some non-negative integer $a$.

To determine which of those 21 fields has cyclic ideal class group of 2-power order we use Proposition 4 and Proposition 20 below.

Proposition 20. Let $M$ be a $C M$-field such that there exists a cyclic quartic $M / K$ with $K \subset M^{+} \subset M$. Let $C_{M}^{(2)}$ and $C_{M^{+}}^{(2)}$ be the 2-Sylow subgroups of $C_{M}$ and $C_{M^{+}}$, respectively.

(1) The three following conditions are equivalent:

(a) $C_{M}^{(2)} / i_{M / M^{+}}\left(C_{M^{+}}^{(2)}\right)$ is non-cyclic.

(b) $\left|C_{M}^{(2)} / i_{M / M^{+}}\left(C_{M^{+}}^{(2)}\right)\right| \geq 4$.

(c) $h_{M}^{-} \equiv 0 \bmod 4$ if $i_{M / M^{+}}$is injective and $h_{M}^{-} \equiv 0 \bmod 2$ otherwise.

If these conditions are verified, then $C_{M}^{(2)}$ is not cyclic. 
(2) If $C_{M}^{(2)} / i_{M / M^{+}}\left(C_{M^{+}}^{(2)}\right)$ is cyclic, then $C_{M}^{(2)}$ and $C_{M^{+}}^{(2)}$ are cyclic. In addition, $M$ satisfies one of the three following conditions :

(a) $Q_{M}=1, i_{M / M^{+}}$is injective and $t_{M} \leq 2$,

(b) $Q_{M}=2$ and $t_{M} \leq 1$,

(c) $i_{M / M^{+}}$is not injective and $t_{M} \leq 1$.

Here $t_{M}$ is the number of prime ideals ramified in $M / M^{+}$.

Proof. See Lemma II.1 and Proposition II.3 in [Gu.

\section{ACKNOWLEDGEMENTS}

We are greatly indebted to S. Louboutin for suggesting the problem and several helpful comments. We were notified after submission of this paper that Yamamura has recently completed the determination of the imaginary abelian number fields with relative class number one and class number $>1$ ([Y2]).

\section{REFERENCES}

[A] S. Arno, The imaginary quadratic fields of class number 4, Acta Arith. 60(1992), 321334. MR 93b:11144

[CK] K. -Y. Chang and S. -H. Kwon, Class number problem for imaginary cyclic number fields, J. Number Theory 73(1998), 318-338. MR 99i:11102

[G] M. -N. Gras, Classes et unités des extensions cycliques réelles de degré 4 de $\mathbb{Q}$, Ann. Inst. Fourier(Grenoble) 29(1979), no 1, XIV, 107-124. MR 81f:12003

[Gu] G. Guerry, Sur la 2-composante du group des classes de certaines extensions cycliques de degré $2^{N}$, J. Number Theory 53(1995), 159-172. MR 96j:11151

[Ha] H. Hasse, Über die Klassenzahl abelscher Zahlkörper, Academie-Verlag, Berlin, 1952. Reprinted with an introduction by J. Martinet: Springer-verlag, Berlin, 1985. MR 14:141a; MR 87j:11122a

$[\mathrm{HH}] \quad$ K. Horie and M. Horie, CM-fields and exponents of their ideal class groups, Acta Arith. 55(1990), 157-170. MR 91k:11098

[Ho] K. Horie, On a ratio between relative class numbers, Math. Z. 211(1992), 505-521. MR 94a:11171

[HY1] M. Hirabayashi and K. Yoshino, Remarks on unit indices of imaginary abelian number fields, Manuscripa Math. 60(1988), 423-436. MR 89e:11068

[HY2] M. Hirabayashi and K. Yoshino, Remarks on unit indices of imaginary abelian number fields II, Mamuscripta Math. 64(1989), 235-251. MR 90j:11115

[HY3] M. Hirabayashi and K. Yoshino, Unit indices of imaginary abelian number fields of type (2,2,2), J. Number Theory, 34(1990), 346-361. MR 91e:11125

[K] T. Kubota, Über den bizylischen biquadratischen Zahlkörper, Nagoya Math. J., 10(1956), 65-85. MR 18:643e

[KT] M. Daberkow, C. Fieker, J. Klüners, M. Pohst, K. Roegner and K. Wildanger, Computational algebra and number theory, KANT V4, J. Symbolic Comp. 24(1997), 267-283. MR 99g:11150

[Lem] F. Lemmermeyer, Ideal class groups of cyclotomic number fields I, Acta Arith. 72. 4(1995), 347-359. MR 96h:11111

[Li] F. J. van der Linden, Class number computations of real abelian number fields, Math. Comp. Vol. 39, No 160(1982), 693-707. MR 84e:12005

[LO] S. Louboutin and R. Okazaki, The class number one problem for some non-abelian normal CM-fields of 2-power degrees, Proc. London Math. Soc. Vol.76, part 3(1998), 523-548. MR 99c: 11138

[LOO] S. Louboutin, R. Okazaki and M. Olivier, The class number one problem for some non-abelian normal CM-fields, Trans. Amer. Math. Soc. 349(1997), 3657-3678. MR 97k:11149

[Lou1] S. Louboutin, CM-fields with cyclic ideal class groups of 2-power orders, J. Number Theory 67(1997), 1-10. MR 98h:11139 
[Lou2] S. Louboutin, Lower bounds for relative class numbers of CM-fields, Proc. Amer. Math. Soc. 120(1994), 425-434. MR 94d:11089

[Lou3] S. Louboutin, Upper bounds on $|L(1, \chi)|$ and application, Canad. J. Math., 50(4),1998, 794-815. MR 99f:11149

[Lou4] S. Louboutin, Determination of all nonquadratic imaginary cyclic number fields of 2power degrees with ideal class groups of exponents $\leq 2$, Math. Comp. Vol. 64, No. 209(1995), 323-340. MR 95c:11124

[Lou5] S. Louboutin, Minoration au point 1 des fonctions $L$ et détermination des corps sextiques abéliens totalement imaginaires principaux, Acta. Arith. 62.2(1992),109-124. MR 93h:11100

[Mu M. R. Murty, An analogue of Artin's conjecture for abelian extensions, J. Number Theory 18(1984), 241-248. MR 85j:11161

[MW] H. L. Montgomery and P.J. Weinberger, Notes on small class numbers, Acta Arith. 24 (1973/74) 529-542. MR 50:9841

[Ok] R. Okazaki, Inclusion of CM-fields and divisibility of relative class numbers, Preprint, 1996, Doshisha Univ.

[PK1] Y.-H. Park and S.-H. Kwon, Determination of all imaginary abelian sextic number fields with class number $\leq 11$, Acta Arith. 82. 1(1997), 27-43. MR 98i:11094

[PK2] Y.-H. Park and S.-H. Kwon, Determination of all non-quadratic imaginary cyclic number fields of 2-power degree with class number $\leq$ 20, Acta Arith. 83. 3(1998), 211-223. MR 99a: 11125

[S1] H. M. Stark, Some effective case of the Brauer-Siegel theorem, Invent. Math. 23(1974), 135-152. MR 49:7218

[S2] H. M. Stark, A complete determination of the complex quadratic fields of class number one, Michigan Math. J. 14(1967), 1-27. MR 36:5102

[S3] H. M. Stark, On complex quadratic fields with class number two, Math. Comp. 29(1975), 289-302. MR 51:5548

[T] T. Tatuzawa, On a theorem of Siegel, Japanese J. Math.21(1951),163-178. MR 14:452c

[W] L. C. Washington, Introduction to cyclotomic fields, GTM 83. 2nd Ed., Springer-Verlag, 1996. MR 97h:11130]

[Y1] K. Yamamura, The determination of imaginary abelian number fields with class number one, Math. Comp. 62(1994), 899-921. MR 94g:11096

[Y2] K. Yamamura, Table of the imaginary abelian number fields with relative class number one and class number $>1$, Preprint(1999).

Department of Mathematics, Korea University, 136-701, Seoul, Korea

E-mail address: jang@semi.korea.ac.kr

Department of Mathematics Education, Korea University, 136-701, Seoul, Korea

E-mail address: shkwon@semi.korea.ac.kr 